\title{
FATIGUE PERFORMANCE OF BITUMINOUS MIXTURES MADE WITH RECYCLED CONCRETE AGGREGATES AND WASTE TIRE RUBBER
}

\author{
A.R. Pasandin ${ }^{1, *}$ and I. Pérez ${ }^{1}$ \\ ${ }^{1}$ Universidade da Coruña, E. T. S. I. Caminos, Canales y Puertos, Campus de Elviña s/n, 15071. A \\ Coruña, Spain \\ ${ }^{*}$ Corresponding author. Tel.: +34-981167000. Fax: $+34-981167170$ \\ E-mail addresses: arodriguezpa@udc.es (A.R.Pasandín),iperez@udc.es (I. Pérez)
}

\begin{abstract}
Fatigue cracking is one of the main hot-mix asphalt (HMA) failure modes. The current laboratory investigation analyses the fatigue performance of HMA made with recycled concrete aggregates (RCA). An HMA type AC 22 bin S made with 0\%, 35\% and $42 \%$ of RCA was tested in the indirect tensile fatigue test (ITFT) device. Three constant stress levels, ranging from $150 \mathrm{kPa}$ to $350 \mathrm{kPa}$ were used. Mixtures were manufactured at the optimum bitumen content using two types of bitumen: a B35/50 penetration grade bitumen and a $10 \%$ waste tire rubber modified bitumen, BC35/50. This investigation demonstrates the beneficial effect on fatigue life of the incorporation of RCA. Additionally, the use of crumb rubber could lead to RCA bituminous mixtures with higher fatigue life in medium traffic roads.
\end{abstract}

Keywords: fatigue life; hot-mix asphalt; recycled concrete aggregates; indirect tensile fatigue test; waste tire rubber 


\section{Introduction}

Hot-mix asphalt (HMA) is a widely used road pavement material. Of the more than 5.2 million $\mathrm{km}$ of the European road and highway network, it is estimated that over $90 \%$ are paved with HMA or other bituminous materials [1]. Similarly, more than $92 \%$ of the more than 4 million km of U.S. highways and roads are paved with asphalt [1].

Transport infrastructures enhance the economic and social development of a region, increase accessibility and help to mitigate territorial imbalances. For all of these reasons, the quality and durability of highways and road networks must be ensured. It is very important to analyse HMA properties and weaknesses carefully.

Fatigue cracking is one of the main HMA failure modes along with rutting and low temperature cracking [2]. Fatigue cracking results in the shortening of pavement life [3] and is directly related to the quality of road asphalt pavements [4]. Fatigue cracking occurs when the asphalt pavement is subjected to repeated traffic loading [5], as a consequence of the passage of vehicle wheels with a loading level lower than the tensile strength of the material [4]. Despite this low loading level, the cumulative effect of repeated traffic loading over time leads to the appearance of fissures. This phenomenon usually occurs in two stages. In the first stage, called "initiation phase", the appearance of micro-cracks (not visible to the naked eye) that reduce the stiffness of the HMA occurs [6]. Later, in a second stage called "propagation", as traffic loads continue, the coalescence of micro-cracks gives way to the formation and growth of macro-cracks [7]. The growth of these macro-cracks begins with stable crack growth and continues with unstable crack growth [8] that finally, at the end of the second phase, leads to the structural failure of the mixture. 
It is necessary to take into account that other mechanisms, such as self-heating or bitumen tixotropy, which are acting in parallel to cause fatigue, can reduce stiffness [6]. These mechanisms are predominant at the beginning of the "initiation" phase [6].

The fatigue structural failure manifests itself at the flexible pavement surface in two main cracking forms:

- When the bituminous layer is thin or medium, the highest tensile stress is at the bottom of the HMA as a result of bending of the bituminous layer when vehicles pass. In this case, fatigue cracking is usually visible as interconnected cracks, known as "alligator" cracking [9] because of the appearance (figure 1a). These cracks may allow water infiltration to reach the base course or the subgrade [4] and lead to the subsequent appearance of other pavement distress, such as potholes. When "alligator" cracking occurs, the classical fatigue approach, that is, bottom-up fatigue cracking, is prevalent: as a consequence of the horizontal tensile strains or stresses induced by the traffic at the bottom of HMA layers, cracks are originated and propagate upward to the surface course [10]. As a result, the loss of stiffness originated by the appearance of cracks finally leads to increased tensile strains at the bottom of the asphalt layers and increased surface deflections [11].

- When the thickness of the bituminous layer is sufficiently high, the most prejudicial stresses are shear stresses originating at the top of the HMA, which are the consequence of the complex contact pressure distribution under the vehicle tire [11]. This causes fatigue of HMA in the form of longitudinal cracking along the wheel path [10] together with small transverse cracks [11] (figure 1b). In this case, the prevalent fatigue mechanism is top-down fatigue cracking: cracks initiate at the HMA surface and progress downwards through 
the bituminous layer. Environmental factors, such as thermal stresses and the existence of damage zones, may accelerate top-down fatigue cracking [12].

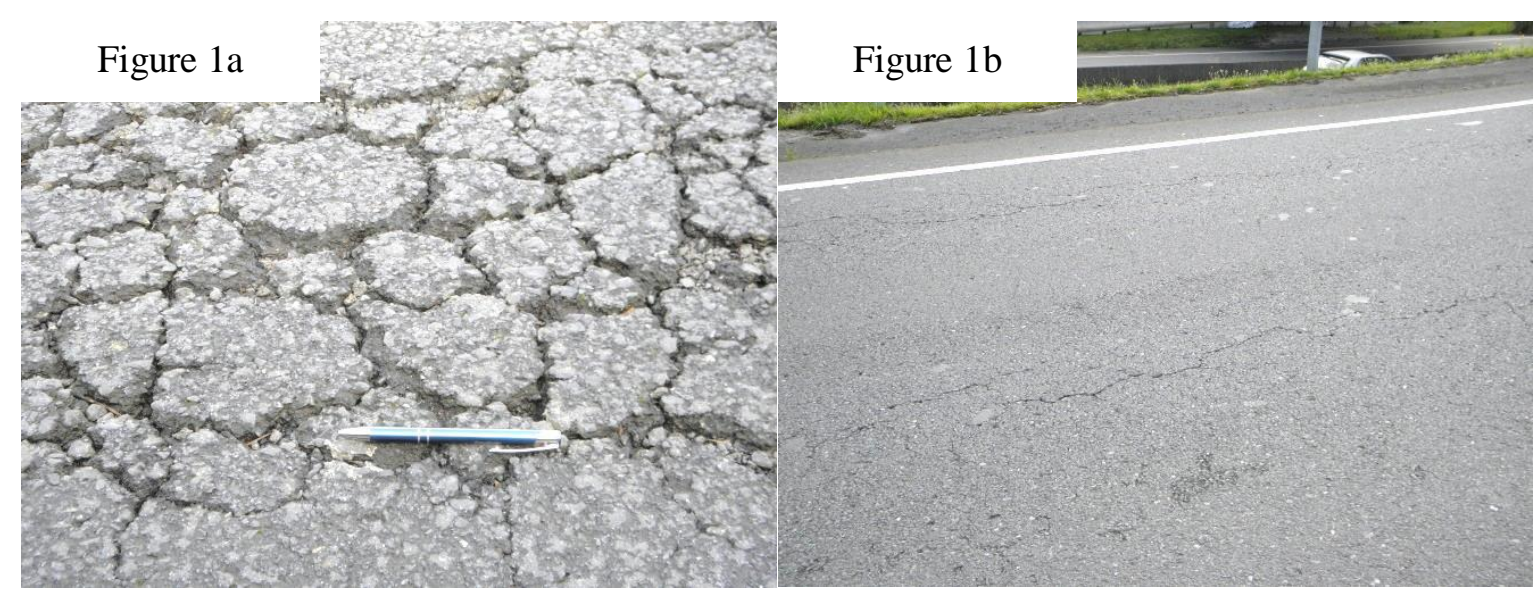

Figure 1. Cracking due to HMA fatigue: a) "Alligator" cracking and b) Longitudinal cracks along the wheel pad.

HMA is typically composed of approximately 95 percent mineral aggregates mixed with 5 percent of bituminous binder [1]. In some cases, the addition of small amounts of other materials such as fibres, may improve some of the bituminous mixture's properties [10].

In view of these percentages, it is clear that the substitution of virgin mineral aggregates with recycled aggregates will have economic and environmental advantages. For these reasons, the use of residues and industrial by-products as recycled aggregates has been an excellent choice over the last few decades. Some of these residues are still being studied. This is the case with recycled concrete aggregates (RCA) from construction and demolition waste (C\&DW).

Some authors have investigated the use of RCA on HMA, with encouraging results [13]. The fatigue resistance of HMA made with RCA is one of the less studied properties. In this regard Chen et al. [14] followed the AASHTO T-321 to analyse the fourpoint bending fatigue life, concluding that the use of RCA as mineral filler in HMA 
produced mixtures with higher fatigue resistance. Pérez et al. $[15,16]$ followed Spanish NLT-350 standard to analyse the three-points bending fatigue life of HMA made with RCA up to $60 \%$, concluding that these mixtures behave similar to conventional ones. Arabani and Azarhoosh [17] used the Nottingham Asphalt Tester to analyse the fatigue life of mixtures made with RCA. They concluded that when RCA is used in the fine fraction, the fatigue life of the mixtures increases, while when RCA is used in the coarse fraction, the fatigue life decreases. Moghadas Nejad et al. [18] used the indirect tensile fatigue test and concluded that the use of up to $100 \%$ RCA improved the fatigue life of the bituminous mixtures. Pasandín and Pérez [19] concluded that mixtures up to $20 \%$ of RCA perform similar to conventional ones, while higher percentages of RCA lead to mixtures with poor fatigue life. Also Pasandín and Pérez [20] stated that mixtures made with RCA coated with bitumen emulsion perform similar to conventional mixtures in terms of fatigue life.

The aim of this investigation is

- Determine whether the use of RCA affects the fatigue resistance of HMA.

- Evaluate whether there is any trend in fatigue life as the RCA percentage in HMA is increased.

- Reach a better understanding of the performance of HMA made with RCA.

To fulfil these objectives, an experimental laboratory investigation was conducted that focused on the fatigue resistance of HMA made with RCA. In the study, an AC 22 bin S made with $0 \%, 35 \%$ and $42 \%$ RCA was tested in the indirect tensile fatigue test (ITFT) device at a constant temperature of $20^{\circ} \mathrm{C}$ at the optimum bitumen content with two types of bitumen: $\mathrm{B} 35 / 50$, penetration grade bitumen, and $\mathrm{BC} 35 / 50$, a waste tire rubber modified bitumen. 


\section{Materials and Methods}

\subsection{Basic Materials}

\subsubsection{Aggregates}

Two aggregates were used in this investigation: natural aggregates and recycled concrete aggregates (RCA) from construction and demolition waste (C\&DW).

The natural aggregates were crushed limestone from a local quarry in Madrid (Spain). RCA were obtained from the demolition of residential buildings and were supplied by a C\&DW recycling plant in Madrid (Spain).

As shown in table 1, RCA display lower bulk specific gravity ( $\rho$ a) than the limestone as well as higher water absorption $\left(\mathrm{W}_{24}\right)$. The cement mortar adhering to the RCA surface, which is more porous and less dense than the aggregates [21], is mainly responsible of this characteristic. This phenomenon is more pronounced in the finest fractions due to the higher percentage of mortar included in this fraction [21].

The sand equivalent (SE) values of the RCA (67\%) and the limestone (59\%) obtained according to UNE-EN 933-8 complied with the Spanish General Technical Specifications for Roads (PG-3) [22], for HMA as a base course material for all heavy traffic categories (SE > 55\%).

The flakiness index (FI) of RCA (8\%) and limestone (15\%) obtained according to UNEEN 933-3 also complies with the PG-3 for all heavy traffic categories (FI $\leq 20 \%)$.

The Los Angeles (LA) abrasion coefficient obtained according to UNE-EN 1097-2 for the limestone $(15 \%)$ also complies with PG-3 (LA $\leq 25 \%)$ for all heavy traffic categories. The LA for the RCA (32\%) is too high and only complies with the PG-3 
(LA $\leq 35 \%$ ) for medium and light heavy traffic categories (Annual Average Daily

Heavy Traffic < 200 vehicles per day).

Table 1. Bulk specific gravity and water absorption of RCA and limestone obtained according to UNE-EN 1097-6.

\begin{tabular}{llll}
\hline \multicolumn{2}{l}{ Aggregate } & RCA & Limestone \\
& & & \\
\hline & $0 / 2 \mathrm{~mm}$ & 2.693 & 2.720 \\
$\mathrm{\rho a}$ & $2 / 4 \mathrm{~mm}$ & 2.675 & 2.709 \\
$\left(\mathrm{~g} / \mathrm{cm}^{3}\right)$ & $4 / 8 \mathrm{~mm}$ & 2.645 & 2.686 \\
& $8 / 16 \mathrm{~mm}$ & 2.630 & 2.688 \\
& $16 / 22.4 \mathrm{~mm}$ & 2.605 & 2.686 \\
\hline & $0 / 2 \mathrm{~mm}$ & 7.467 & 1.404 \\
$\mathrm{WA}_{24}$ & $2 / 4 \mathrm{~mm}$ & 5.772 & 2.351 \\
$(\%)$ & $4 / 8 \mathrm{~mm}$ & 5.065 & 1.473 \\
& $8 / 16 \mathrm{~mm}$ & 4.376 & 1.671 \\
& $16 / 22.4 \mathrm{~mm}$ & 4.088 & 1.631 \\
\hline
\end{tabular}

\subsubsection{Bitumen}

The asphalt binder used in this investigation was a penetration grade bitumen B35/50 typically used in HMA manufacture in Spain. The same bitumen, modified with $10 \%$ by weight waste tire rubber, BC35/50, was also used. Its main properties are summarized in Table 2.

Table 2. Basic properties of bitumen.

\begin{tabular}{|c|c|c|c|c|}
\hline Test & Standard & B35/50 & BC35/50 & $\begin{array}{c}\text { Specification } \\
{[23]} \\
\end{array}$ \\
\hline $\begin{array}{l}\text { Penetration }\left(100 \mathrm{~g}, 5 \mathrm{~s}, 25^{\circ} \mathrm{C}\right), 0.1 \\
\mathrm{~mm}\end{array}$ & $\begin{array}{l}\text { UNE-EN } \\
1426\end{array}$ & 41 & 38 & $35-50$ \\
\hline Softening point, ${ }^{\circ} \mathrm{C}$ & $\begin{array}{l}\text { UNE-EN } \\
1427\end{array}$ & 53 & 64 & $\begin{array}{c}50-58 \text { for } \\
\text { B35/50 } \\
\geq 58 \text { for } \\
\text { BC } 35 / 50\end{array}$ \\
\hline
\end{tabular}




\subsection{Specimen Preparation}

A hot-mix asphalt type AC 22 bin $\mathrm{S}$ has been selected for this investigation. Figure 2 shows the grain size distribution of the mixture that is included and the limits specified by the PG-3 [22].

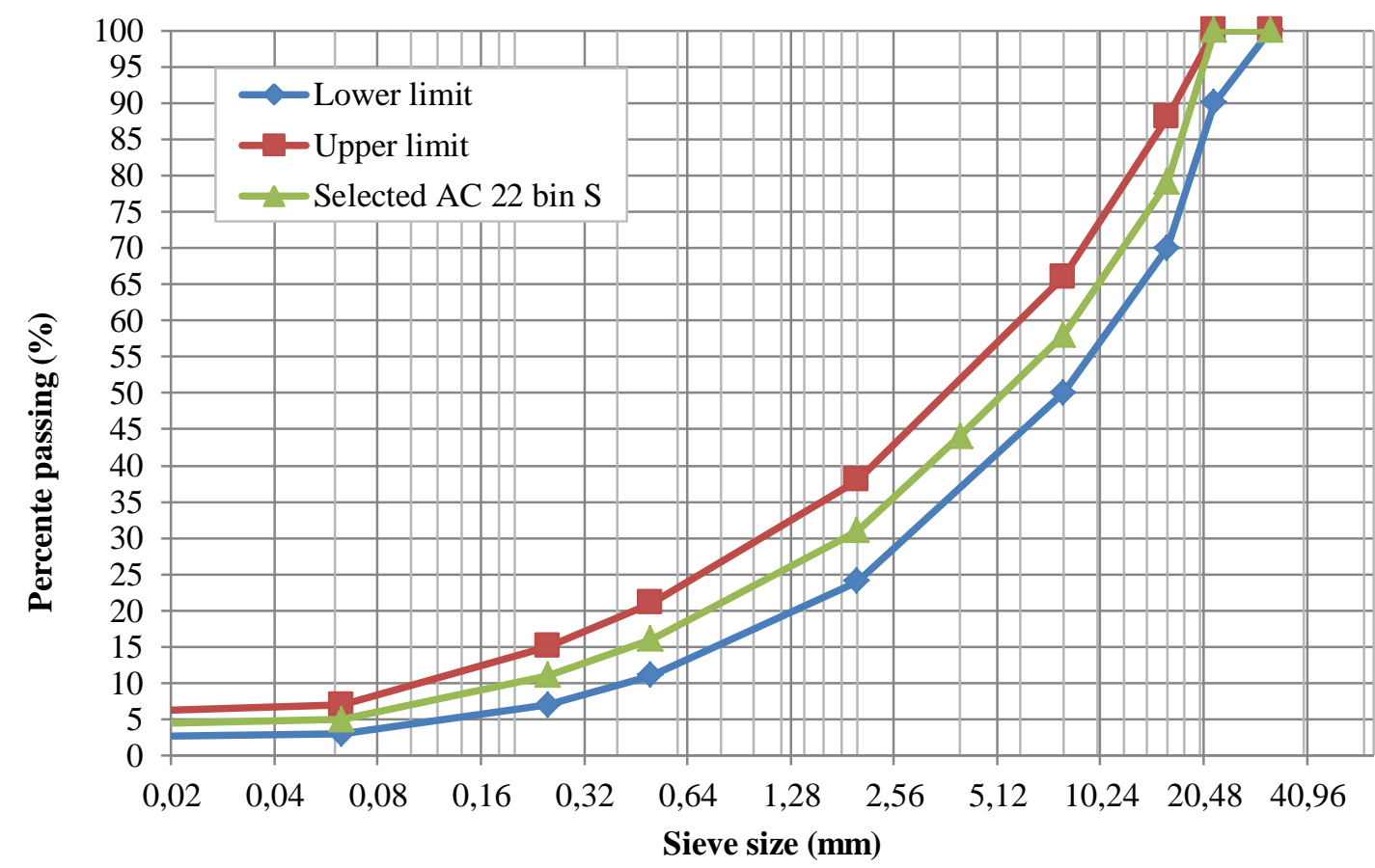

Figure 2. Grain size distribution of AC 22 bin S.

Specimens with $0 \%, 35 \%$ and $42 \%$ of RCA were studied. The specimens were manufactured at their optimum bitumen content. To obtain this optimum bitumen content, volumetric properties according to UNE-EN 12697-8 and water sensitivity according to UNE-EN 12697-12 were analysed. The optimum bitumen content for each bitumen type and each RCA percentage is the minimum that displays air voids ranging from $4 \%$ to $7 \%$ and achieves a minimum indirect tensile stress ratio of $80 \%$, according to the PG-3 [22]. Also, according to the PG-3, the optimum bitumen content must be equal or higher than $4.0 \%$. 
Table 3 shows the optimum bitumen content used in this investigation for each mixture, their air voids (Va) and their indirect tensile stress ratio (TSR).

Table 3. Volumetric properties and TSR at the optimum bitumen content.

\begin{tabular}{|c|c|c|c|c|}
\hline $\mathrm{RCA}(\%$ & & $\begin{array}{l}\text { HMA with } \\
\text { B35/50 }\end{array}$ & $\begin{array}{l}\text { HMA with } \\
\text { BC35/50 }\end{array}$ & $\begin{array}{l}\text { PG-3 limits } \\
{[22]}\end{array}$ \\
\hline \multirow{4}{*}{$0 \%$} & $\mathrm{Va}(\%)$ & 4.49 & 6.09 & $4-7$ \\
\hline & TSR (\%) & 80.24 & 80.09 & $\geq 80$ \\
\hline & Optimum & & & minimum: \\
\hline & $\begin{array}{l}\text { bitumen content } \\
(\%)\end{array}$ & 4.75 & 5.0 & $4.0 \%$ \\
\hline \multirow{4}{*}{$35 \%$} & $\mathrm{Va}(\%)$ & 4.76 & 6.42 & $4-7$ \\
\hline & $\operatorname{TSR}(\%)$ & 83.23 & 85.85 & $\geq 80$ \\
\hline & Optimum & & & minimum: \\
\hline & $\begin{array}{l}\text { bitumen content } \\
(\%)\end{array}$ & 5.25 & 5.50 & $4.0 \%$ \\
\hline \multirow{4}{*}{$42 \%$} & $\mathrm{Va}(\%)$ & 5.13 & 4.19 & $4-7$ \\
\hline & TSR (\%) & 80.95 & 80.30 & \multirow{3}{*}{$\begin{array}{l}\geq 80 \\
\text { minimum: } \\
4.0 \%\end{array}$} \\
\hline & Optimum & & & \\
\hline & $\begin{array}{l}\text { bitumen content } \\
(\%)\end{array}$ & 5.75 & 6.0 & \\
\hline
\end{tabular}

\subsection{Experimental Procedure}

Indirect Tensile Fatigue Test (ITFT) according to UNE-EN 12697-24 controlled stress procedure was conducted. The controlled procedure is preferred to the controlled strain method because of its better correspondence with field conditions [24]. In this test, pulse repetition simulates traffic passing on the bituminous mixture.

The Marshall specimens required for the test (Figure 3a) were manufactured in accordance with Spanish NLT-159/86 [25], compacting them with 75 blows per side of the Marshall hammer. These cylindrical Marshall specimens were manufactured at the optimum bitumen content (table 3 ) for each RCA percentage $(0 \%, 35 \%$ and $42 \%)$.

In Cooper's pneumatic press NU14 (figure 3b), each of these series were tested in a temperature controlled cabinet at a reference temperature of $20^{\circ} \mathrm{C}$. Three constant stress 
levels, typically ranging from $150 \mathrm{kPa}$ to $350 \mathrm{kPa}$ were used. A minimum of three samples for each of the three stress levels were tested, so that a minimum of 10 samples were tested for each RCA content. Each cylindrical Marshall sample was subjected to repeated haversine loads along a vertical diameter until reaching failure. The load time was $0.1 \mathrm{~s}$ and the rest time was $0.4 \mathrm{~s}$. During the 10 first cycles, the initial deformation must range between 100 to $400 \mu \varepsilon$. If the initial deformation is out of this range, the load must be adjusted.

Figure $3 \mathrm{a}$
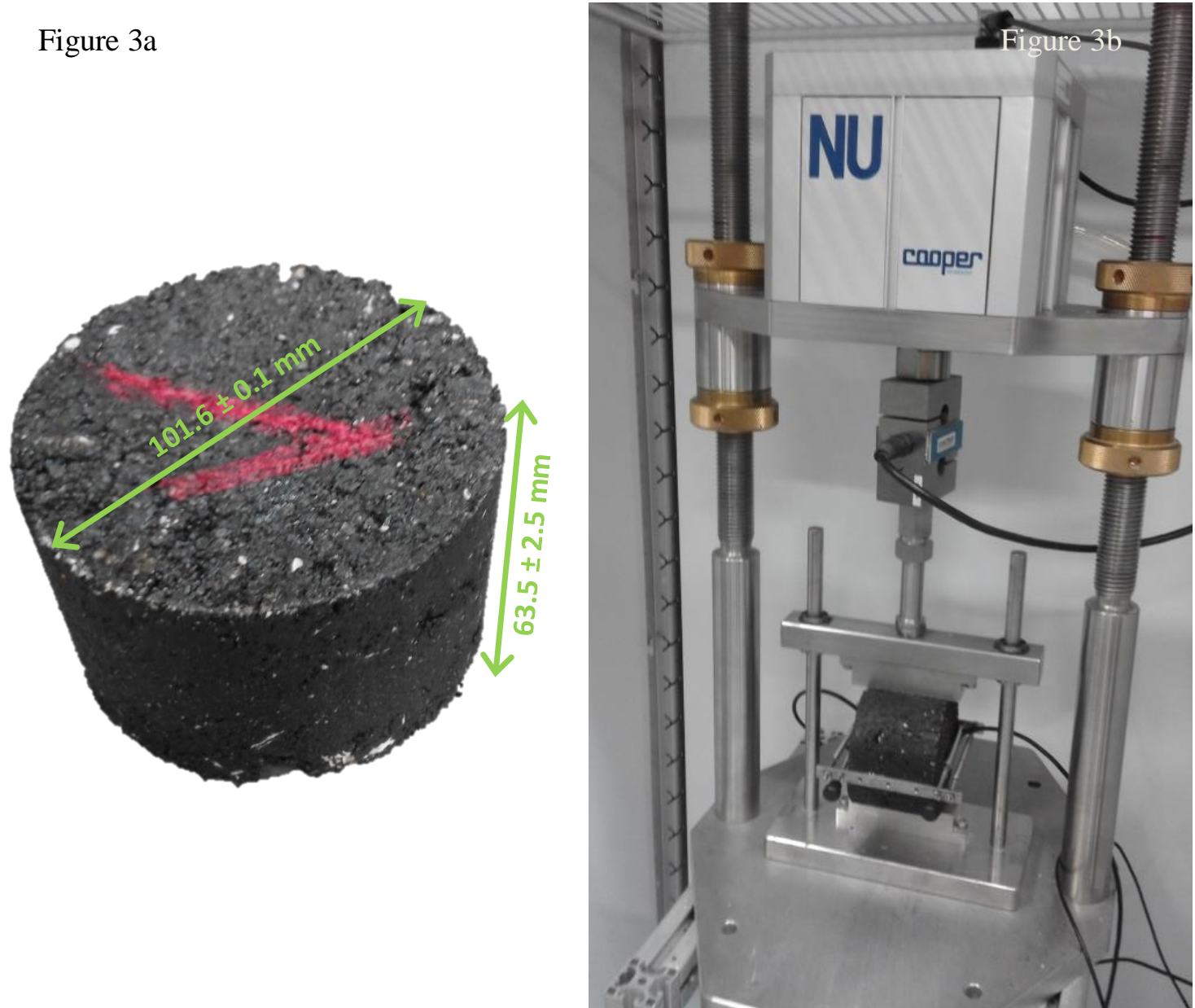

Figure 3: a) Marshall cylindrical specimen and b) Cooper NU14 servo-pneumatic machine.

To obtain the fatigue laws for fatigue life prediction, the Whöler equation was used:

$$
\varepsilon_{0}=k . N f^{-n}
$$


where $\mathrm{Nf}=$ the number of load cycles until fatigue failure; $\mathrm{k}$ and $\mathrm{n}=$ material constants obtained from the ITFT and $\varepsilon_{0}=$ the initial tensile horizontal strain at the centre of the sample in $\mu \varepsilon$.

As a result of ITFT the stiffness of the bituminous mixture can be obtained, as follows:

$S_{m i x}=\frac{\sigma_{S}}{\varepsilon_{S}} .(1+3 v)$

where Smix $=$ stiffness module of the bituminous mixture $(\mathrm{MPa}) ; \sigma_{\mathrm{s}}=$ maximun horizontal stress at the centre of the sample $(\mathrm{MPa}) ; \varepsilon_{\mathrm{s}}=$ maximum horizontal strain at the centre of the sample $(\mu \mathrm{m} / \mathrm{m}) ; v=$ Poisson coefficient (a constant value of 0.35 was assumed).

\section{Results and Discussion}

\subsection{Fatigue failure characteristics}

During ITFT, a combination of fatigue and permanent deformation mechanisms may occur [26]. In controlled stress tests, two failure criteria are usually employed in ITFT in order to determine fatigue life [27]:

- The total number of load cycles to complete splitting of the specimen (Figure 4).

In this case, the fatigue mechanism is prevalent and the tensile stress acting perpendicular to the loaded plane is mainly responsible of the failure [28].

- The total number of cycles to reach the $10 \%$ vertical deformation of the specimen along the vertical plane. In this case, the permanent deformation mechanism is prevalent. 


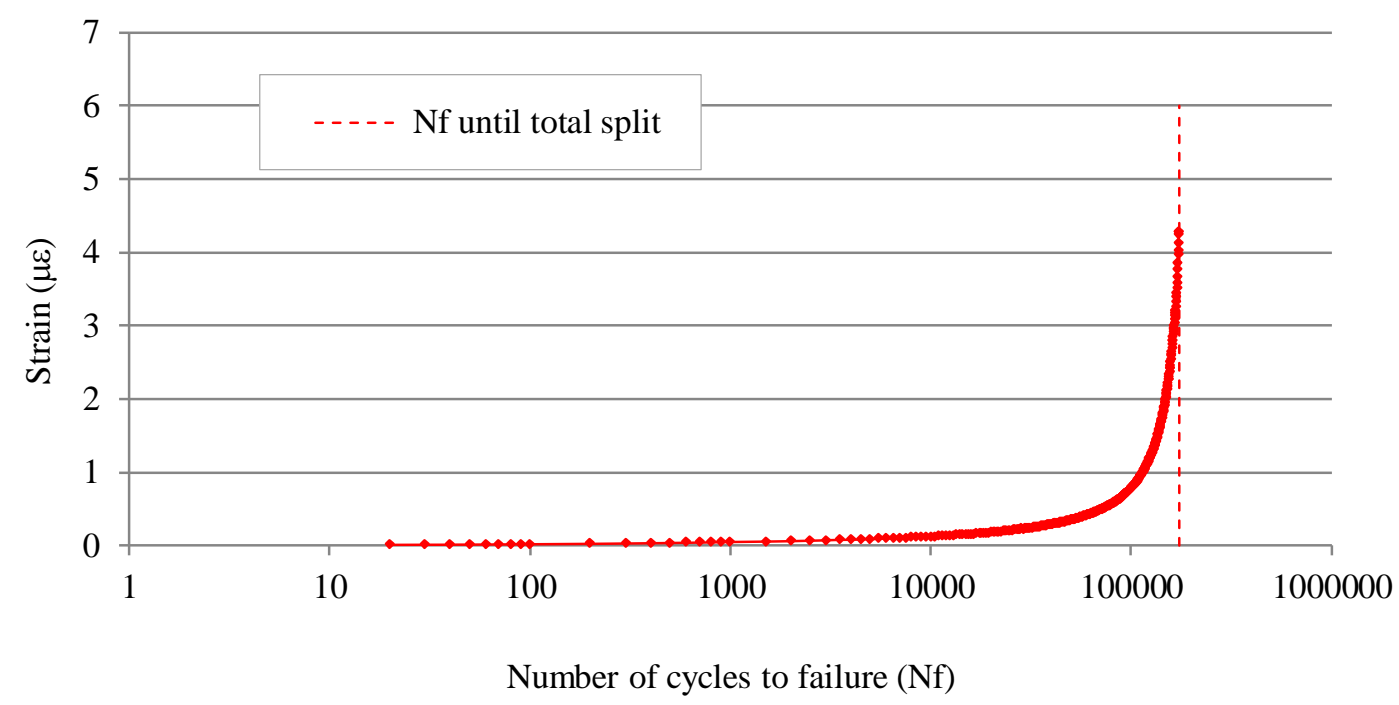

Figure 4. Number of cycles to total structural failure. Mixture made with $0 \%$ RCA and BC35/50.

Of the two failure mechanisms considered, the first has always been given in the present investigation (Figure 5), independently of the RCA percentage and whether the bitumen was $\mathrm{B} 35 / 50$ or $\mathrm{BC} 35 / 50$. Therefore, the mechanism of fatigue prevails over that of permanent deformation.

In addition, this kind of failure can cause different split patterns such as ideal strip, localized crushing failure, double cleft failure, single cleft failure and triple cleft failure [28]. As shown in Figure 5, the single cleft failure pattern was produced in all tested samples. 
Figure 5a
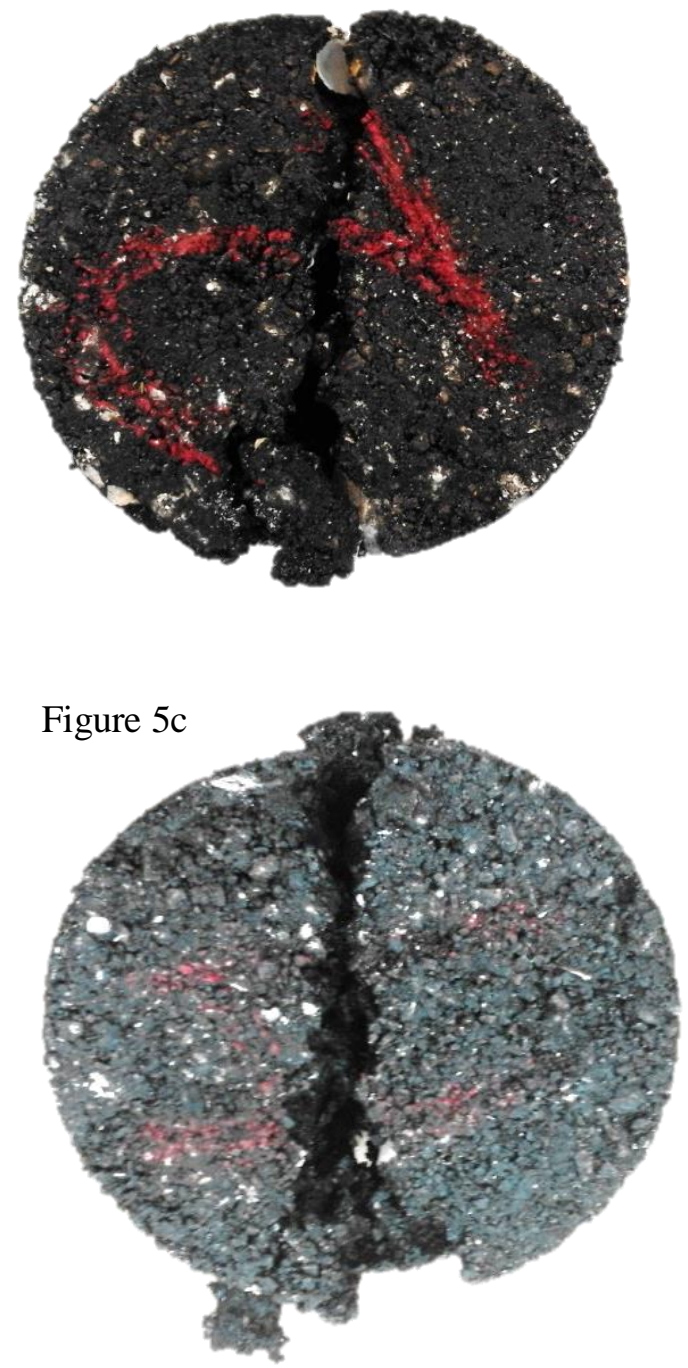

Figure $5 b$

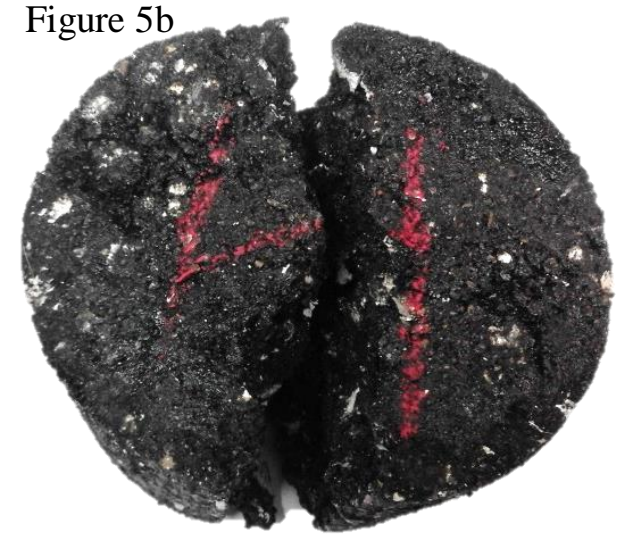

Figure 5d

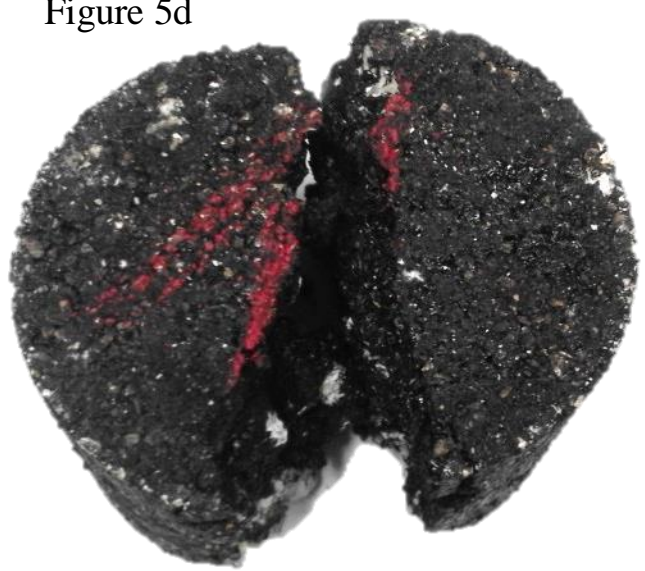

Figure 5: a) 35\% RCA with B35/50, b) $42 \%$ RCA with B35/50, c) $35 \%$ of RCA with BC35/50 and d) $42 \%$ of RCA with BC35/50.

No different split patterns were observed due to the RCA percentage or the type of bitumen. This conclusion is consistent with that stated in the technical literature, since the type of loading strip is primarily responsible for the split pattern [28].

\subsection{Stiffness}

Figure 6 shows the initial stiffness of the mixture (at 100th cycle) versus the initial micro-strain (at $10^{\text {th }}$ cycle) [27] for all the tested mixtures. 


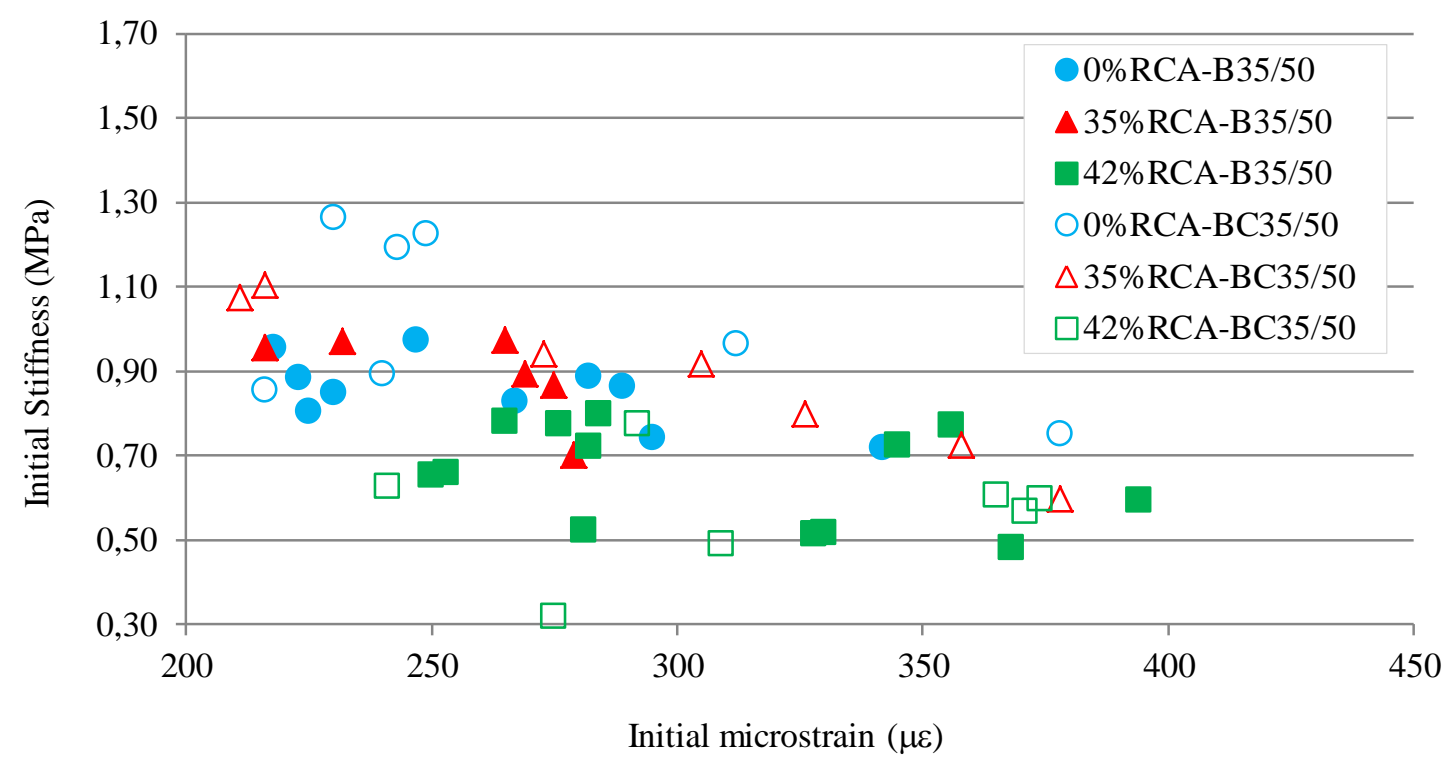

Figure 6. Initial stiffness of the mixture vs initial micro-strain for mixtures made with $0 \%, 35 \%$ and $42 \%$ RCA with B35/50 and BC35/50.

Figure 6 shows that as the initial micro-strain increases, the initial stiffness slightly decreases in all cases. It was an expected result because the ITFT was conducted with the controlled stress procedure.

Figure 6, shows the influence of the type of bitumen, that is, whether the bitumen is modified with waste tire rubber or not. Bituminous mixtures made with $0 \%$ RCA and BC35/50 display higher initial stiffness than mixtures made with 0\% RCA and B35/50. It can be clearly stated that the bitumen modified with waste tire rubber leads to bituminous mixtures with higher stiffness. This is probably due to the increased stiffness of the binders modified with waste tire rubber [29].

When RCA is added to the mixtures (35\% RCA and 42\% RCA) there is almost no difference between the mixtures made with B35/50 and those made with BC35/50. Thus, it can be stated that the RCA influence on the stiffness is higher than the influence of the use of waste tire rubber. 
Additionally, in figure 6, it can be seen that a higher RCA percentage leads to lower initial stiffness for all initial micro-strain levels and for all kinds of tested bitumen. The mortar attached to the RCA surface, which is porous and weak, could explain this behaviour. The higher bitumen consumption as the RCA percentage grows, could also explain this trend. There is only one exception to this performance, mixtures made with $0 \%$ RCA and B35/50 display similar initial stiffness results as those obtained by the mixtures made with $35 \%$ RCA and $\mathrm{B} 35 / 50$ or BC35/50. As above, when no RCA is added to the mixtures, the influence of the bitumen is more noticeable. The absorptive nature of RCA, which is more porous and weak than natural aggregate, may be responsible of this performance.

In addition, in Figure 6, when the mixtures are made with BC35/50, the initial stiffness differences between mixtures with $0 \%, 35 \%$ and $42 \%$ RCA for all of the initial microstrain ranges are higher than the initial stiffness differences for mixtures made with $\mathrm{B} 35 / 50$. This finding is probably observed because the B35/50 is more easily absorbed by the pores of the RCA than the $\mathrm{BC} 35 / 50$, which has higher viscosity.

\subsection{Fatigue life}

Fatigue life is defined as the number of load cycles until failure occurs (Nf) in bituminous mixtures and represents the ability of the mixture to withstand the traffic cyclical loads [30]. Fatigue life is related to service life in such a way that higher fatigue life leads to higher service life [31].

Figure 7 shows the initial horizontal strain versus the number of cycles to failure (total split of the sample) at $20^{\circ} \mathrm{C}$ on a logarithmic scale for the tested mixtures (N-S plot). This figure also includes the fatigue life law equations and the correlation coefficient $\left(\mathrm{R}^{2}\right)$. As seen in Figure 7, this coefficient ranges from 0.8516 to 0.9546 which indicates 
a good statistical relationship between the results obtained to determine each fatigue law.

As can be clearly seen in Figure 7, the higher the percentage of RCA is, the longer the fatigue life of the bituminous mixture is. It was an expected result because, as said above, as RCA percentage grows the stiffness of the mixture decreases. Thus, it can be clearly stated that RCA is beneficial for the fatigue life of the bituminous mixtures. Also, figure 7 shows that bituminous mixtures made with B35/50 displayed similar slopes in their fatigue life laws regardless of the RCA percentage used. The same applies to mixtures made with $\mathrm{BC} 35 / 50$. This shows the strong influence of the type of bitumen in the fatigue life of hot-mix asphalt.

In this regard, mixtures made with $\mathrm{BC} 35 / 50$ displayed more pronounced slopes than mixtures made with B35/50. For this reason, for lower initial micro-strains, mixtures made with $\mathrm{BC} 35 / 50$ have lower fatigue life than mixtures made with B35/50. For higher initial micro-strain, mixtures made with BC35/50 present higher fatigue life.

The initial micro-strain from which this change of behaviour occurs varies depending on the RCA percentage. For $0 \%$ RCA, the initial micro-strain of $303.1 \mu \mathrm{m}$, is the limit for this change of performance. However, for 35\% RCA the initial micro-strain is 226,6 $\mu \mathrm{m}$ and in the case of $42 \%$ RCA the initial micro-strain is $264,7 \mu \mathrm{m}$. As seen, there is no correlation between the initial micro-strain limit and the RCA percentage. 


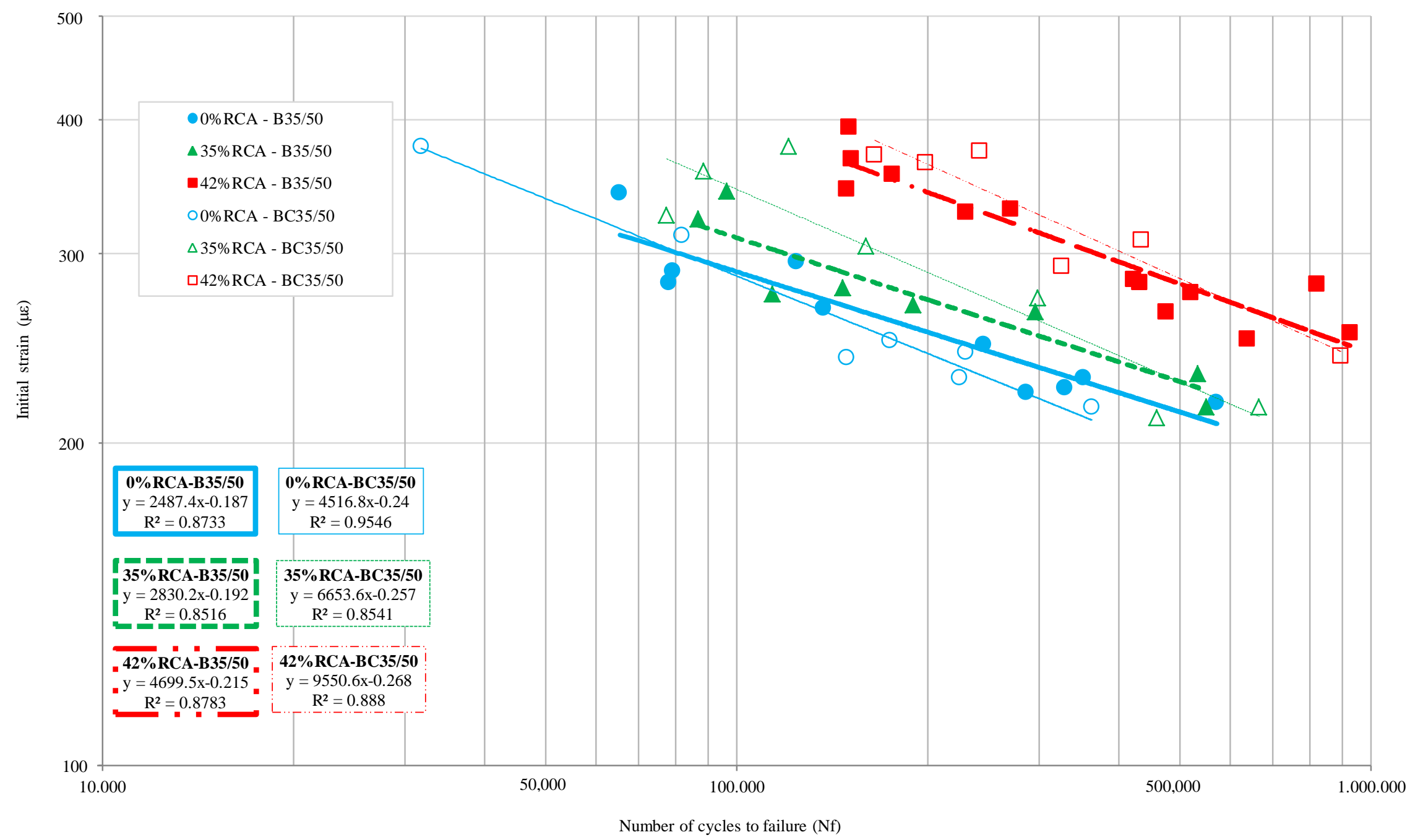

Figure 7. N-S plot and fatigue life laws for mixtures made with $0 \%, 35 \%$ and $42 \% \mathrm{RCA}$ with $\mathrm{B35} / 50$ and $\mathrm{BC} 35 / 50$. 
As said before, Figure 7 shows that the differences between fatigue law equations are mainly due to the RCA percentage used and to the use of $\mathrm{B} 35 / 50$ or $\mathrm{BC} 35 / 50$.

Nevertheless, it must be noted that the RCA percentage produces more pronounced changes in fatigue laws than the type of bitumen used. It was an expected result as seen in section 3.2.

\section{Conclusions}

This paper analyses fatigue performance of HMA made with RCA. The research focused on the fatigue resistance of an $\mathrm{AC} 22$ bin $\mathrm{S}$ manufactured with $0 \%, 35 \%$ and $42 \%$ RCA in place of natural limestone aggregates. In addition, two types of bitumen, $\mathrm{B} 35 / 50$ and $\mathrm{BC} 35 / 50$, were used since waste tire rubber could affect the fatigue life of such mixtures. From the investigation the following conclusions were drawn:

- In this type of mixture, during the ITFT, the fatigue mechanism is prevalent versus the permanent deformation mechanism, independent of the RCA percentage and the use of waste tire rubber as bitumen modifier.

- The higher the percentage of RCA is (up to $42 \%$ ), the longer the fatigue life of the bituminous mixture is. Thus, the introduction of this waste material not only is not detrimental to the fatigue performance of HMA but is beneficial. The lower stiffness displayed by mixtures made with higher RCA percentage, could explain this trend.

- The use of waste tire rubber as a bitumen modifier clearly affects the fatigue performance of HMA made with RCA.

- For lower initial micro-strains, mixtures made with BC35/50 have lower fatigue life than mixtures made with B35/50. For higher initial micro-strains, mixtures made with BC35/50 have higher fatigue life. 
- HMA made with RCA are only suitable for light and medium traffic categories due to the low resistance to the fragmentation of RCA.

- Taking into account the two previous conclusions, it can be stated that for medium traffic roads, the use of waste tire rubber could improve the fatigue resistance of HMA made with RCA. In contrast, for light traffic categories, the fatigue life of mixtures made with RCA could be shortened if waste tire rubber is used as bitumen modifier.

In view of the results, the use of RCA and waste tire rubber in HMA is encouraging, particularly when used in medium traffic roads. Nevertheless, further investigation is warranted.

\section{Acknowledgements}

The authors wish to acknowledge the Spanish Ministry of Education and Science for sponsoring this research through Project BIA2013-47987-C3-2-R.

The authors would also like to thank Tec-Rec for generously donating the RCA and Monte Orusco quarry (Extracción de áridos Sierra Negra, S.L.) for generously donating the limestone aggregate.

\section{References}

[1].National Asphalt Pavement Association. (2011). The Asphalt Paving Industry, A Global Perspective.

[2].Saboo, N., Das, B. P. and Kumar, P. (2016). New phenomenological approach for modeling fatigue life of asphalt mixes. Construction and Building Materials, 121, 134-142. http://dx.doi.org/10.1016/j.conbuildmat.2016.05.147. 
[3].Moghaddam, T. B., Karim, M. R. and Abdelaziz, M. (2011). A review on fatigue and rutting performance of asphalt mixes. Scientific Research and Essays, 6(4), 670-682.

[4].Tigdemir, M., Karasahin, M. and Sen, Z. (2002). Investigation of fatigue behaviour of asphalt concrete pavements with fuzzy-logic approach. International Journal of Fatigue, 24 (8), 903-910. http://dx.doi.org/10.1016/S0142-1123(01)00183-9.

[5].Mannan, U. A., Islam, M. R. and Tarefder, R. A. (2015). Effects of recycled asphalt pavements on the fatigue life of asphalt under different strain levels and loading frequencies. International Journal of Fatigue, 78, 72-80. http://dx.doi.org/10.1016/j.ijfatigue.2015.04.004.

[6].Di Benedetto, H., De La Roche, C., Baaj, H., Pronk, A., Lundström, R. (2004). Fatigue of bituminous mixtures. Materials and structures, 37(3), 202-216. http://dx.doi.org/10.1007/BF02481620.

[7].Füssl, J. and Lackner, R. (2011). Multiscale fatigue model for bituminous mixtures. International Journal of Fatigue, 33(11), 1435-1450. http://dx.doi.org/10.1016/j.ijfatigue.2011.05.014.

[8].Liang, R. Y. and Zhou, J. (1997). Prediction of fatigue life of asphalt concrete beams. International Journal of Fatigue, 19(2), 117-124. http://dx.doi.org/10.1016/S0142-1123(96)00066-7.

[9].Prowell, B., Brown, E. R., Anderson, R. M., Daniel, J. S., Von Quintus, H., Shen, S. and Maghsoodloo, S. (2010). NCHRP Report 646: Validating the fatigue endurance limit for hot mix asphalt. Transportation Research Board of the National Academies, Washington, DC. 
[10]. European Commission. Directorate General Transport (1999). COST 333.

Development of New Bituminous Pavement Design Methods. Final Report of the Action. Luxembourg (Brussels).

[11]. Molenaar, A. A. A. (2004). Bottom-up fatigue cracking: myth or reality. In The 5th RILEM Conference: Cracking in Pavement Mitigation, Risk Assessment and Prevention, Limoges, France (p. 275).

[12]. Roque, R., Zou, J., Kim, Y. R., Beak, C., Thirunavukkarasu, S., Underwood, B. S. and Guddati, M. N. (2010). Top-down cracking of HMA layers: Models for initiation and propagation (NCHRP Web-Only Document 162). National Cooperative Highway Research Program. Transportation Research Board of the National Academies.

[13].Pasandín, A.R. and Pérez, I. (2015). Overview of bituminous mixtures made with recycled concrete aggregates. Construction and Building Materials, 74, 151-161. http://dx.doi.org/10.1016/j.conbuildmat.2014.10.035.

[14]. Chen, M., Lin, J. and Wu, S. (2011). Potential of recycled fine aggregates powder as filler in asphalt mixture. Construction and Building Materials, 25(10), 3909-3914. http://dx.doi.org/10.1016/j.conbuildmat.2011.04.022.

[15].Pérez, I., Toledano, M., Gallego, J. and Taibo, J. (2007). Mechanical properties of hot mix asphalt made with recycled aggregates from reclaimed construction and demolition debris. Materiales de Construcción, 57(285), 17-29.

[16].Pérez, I., Gallego, J., Toledano, M. and Taibo, J. (2010, December). Asphalt mixtures with construction and demolition debris. In Proceedings of the Institution of Civil Engineers-Transport (Vol. 163, No. 4, pp. 165-174). Thomas Telford Ltd. http://dx.doi.org/10.1680/tran.2010.163.4.165. 
[17]. Arabani, M. and Azarhoosh, A.R. (2012). The effect of recycled concrete aggregate and steel slag on the dynamic properties of asphalt mixtures. Construction and Building Materials, 35, 1-7. http://dx.doi.org/10.1016/j.conbuildmat.2012.02.036.

[18]. Moghadas Nejad, F.; Azarhoosh, A.R. and Hamedi, G.H. (2013). The effects of using recycled concrete on fatigue behavior of hot mix asphalt, Journal of Civil Engineering and Management 19 (Supplement 1): S61-S68. http://dx.doi.org/10.3846/13923730.2013.801892.

[19].Pasandín, A.R. and Pérez, I. (2013). Laboratory evaluation of hot-mix asphalt containing construction and demolition waste. Construction and Building Materials, 43, 497-505. http://dx.doi.org/10.1016/j.conbuildmat.2013.02.052

[20].Pasandín, A.R. and Pérez, I. (2014). Mechanical properties of hot-mix asphalt made with recycled concrete aggregates coated with bitumen emulsion. Construction and Building Materials, 55, 350-358. http://dx.doi.org/10.1016/j.conbuildmat.2014.01.053.

[21]. De Juan, M.S. and Gutiérrez, P.A. (2009). Study on the influence of attached mortar content on the properties of recycled concrete aggregate. Construction and Building Materials, 23 (2), 872-877. http://dx.doi.org/10.1016/j.conbuildmat.2008.04.012.

[22]. MFOM (2015). Ministry of Public Works. Article 542 (Asphalt Concrete) of the General Technical Specifications for Road and Bridge Works (PG3) from the Spanish Ministry of Public Works. Madrid, Spain (in Spanish).

[23].Public Works and Transportation Ministry (2007). OC 21/2007 on the use and specifications that must met the binders and the bituminous mixtures incorporating waste tire rubber. In Spanish. 
[24]. Pell PS. Characterization of fatigue behavior. Highway research board special report 140. In: Proceedings of a symposium on structural design of asphalt concrete pavements to prevent fatigue cracking. Washington; 1973: 49-64.

[25]. MOPT (2002). Public Works and Transportation Ministry. NLT Standards. Road Test. General Directorate of Highways, second edition, Madrid, Spain (in Spanish).

[26]. Cocurullo A., Airey G.D., Collop A.C., Sangiorgi C. (2008). Indirect Tensile versus Two-point Bending Fatigue Testing. Proceedings of the ICE - Transport; 161 (4): 207-220. http://dx.doi.org/10.1680/tran.2008.161.4.207.

[27]. Cooper Technology (2010). CRT-UTM-NU. Servo Pneumatic Universal Testing Machine. User Manual.

[28]. Kennedy, T.W. and Hudson, W.R. (1973). Tensile Properties of Subbases for Use in Rigid Pavement Design. Center for Highway Research, University of Texas at Austin. Research Report Number 98-14F. Research Project 3-8-66-98.

[29]. Rodríguez-Alloza, A. M., Gallego, J., and Giuliani, F. (2017). Complex shear modulus and phase angle of crumb rubber modified binders containing organic warm mix asphalt additives. Materials and Structures, 50:77, 1-9. http://dx.doi.org/10.1617/s11527-016-0950-1.

[30]. Muniandy, R., Akhir, N. A.B.C.M., Hassim, S. and Moazami, D. (2014). Laboratory fatigue evaluation of modified and unmodified asphalt binders in stone mastic asphalt mixtures using a newly developed crack meander technique. International Journal of Fatigue, 59, 1-8. http://dx.doi.org/10.1016/j.ijfatigue.2013.08.021.

[31].Guo, Q., Li, L., Cheng, Y., Jiao, Y. and Xu, C. (2015). Laboratory evaluation on performance of diatomite and glass fiber compound modified asphalt 
mixture. Materials \& Design (1980-2015), 66, 51-59.

http://dx.doi.org/10.1016/j.matdes.2014.10.033. 